\title{
Прогнозирование исходов
}

при лабораторно верифицированном

COVID-19 по данным компьютерной

томографии органов грудной клетки:

ретроспективный анализ 38051 пациента

\author{
(C) С.П. Морозов, В.Ю. Чернина, И.А. Блохин, В.А. Гомболевский
}

ГБУЗ «Научно-практический клинический центр диагностики и телемедицинских технологий Департамента здравоохранения города Москвы», Москва, Российская Федерация

Обоснование. В условиях сложившейся эпидемиологической ситуации компьютерная томография органов грудной клетки (КТ ОГК) играет важную роль в диагностике заболевания. Клинические и КТ-данные позволяют врачам в короткие сроки установить вероятность наличия и прогноз у пациентов с COVID-19.

Цель — прогнозирование исходов у лабораторно верифицированных больных COVID-19 по данным КT ОГК с помощью полуколичественной визуальной шкалы степени поражения лёгочной паренхимы (шкала КT0-КТ4).

Материал и методы. Выполнен ретроспективный анализ выгрузки историй болезни из Единого медицинского информационного-аналитического сервиса (ЕМИАС) и протоколов из Единого радиологического информационного сервиса (ЕРИС) в период с 01.03.2020 по 30.07.2020. В исследование включены истории болезней пациентов с диагнозом U07.1 по МКБ-10 (лабораторно верифицированная коронавирусная инфекция), которым с 1 марта по 30 июля 2020 г. включительно проведена КТ ОГК по направлению врача-терапевта при подозрении на внебольничную пневмонию, вызванную COVID-19; максимально допустимый срок между лабораторной верификацией и KT ОГК — не более 5 дней. Срок наблюдения за каждым пациентом - не менее 30 сут от даты проведения КТ. Исследования были выполнены в 48 медицинских организациях, оказывающих первичную медицинскую помощь взрослому населению Москвы. Не вошли в исследование пациенты, у которых результаты теста полимеразной цепной реакции на COVID-19 были отрицательными к 30.07.2020. Шкала KTO-KT4 рекомендована к применению в Российской Федерации для оценки объёма поражения паренхимы лёгкого при подозрении на COVID-19.

Результаты. Итоговый объём выборки - 38051 пациент. По результатам исследования выявлено, что для категории КТ4 риск смерти выше в 3 раза по сравнению с категорией КТО. По кривым Каплана-Мейера для анализа выживаемости доля выживших пациентов в категории КТ3 почти в 3 раза ниже (HR =2,94), чем в категориях КТ0-КТ2. Кроме того, установлено, что чем выше исходная категория КТ, тем ниже риск ухудшения. Время до госпитализации снижалось при увеличении категории по данным КТ ОГК.

Заключение. Визуальная шкала КТО-КТ4 может быть использована в качестве предиктора исходов (госпитализаций и летальных исходов) у пациентов, которым при подозрении на COVID-19 выполнена KT ОГК на базе первичного звена здравоохранения.

Ключевые слова: COVID-19; внебольничная пневмония; компьютерная томография.

\section{Как цитировать}

Морозов С.П., Чернина В.Ю., Блохин И.А., Гомболевский В.А. Прогнозирование исходов при лабораторно верифицированном COVID-19 по данным компьютерной томографии органов грудной клетки: ретроспективный анализ 38051 пациента // Digital Diagnostics. 2020;1(1):27-36. DOl: https://doi.org/10.17816/DD46791 


\title{
Chest computed tomography for outcome prediction in laboratory-confirmed COVID-19: A retrospective analysis of 38,051 cases
}

\author{
(c) Sergey P. Morozov, Valeria Yu. Chernina, Ivan A. Blokhin, Victor A. Gombolevskiy
}

Research and Practical Clinical Center for Diagnostics and Telemedicine Technologies of the Moscow Health Care Department, Moscow, Russian Federation

BACKGROUND: In the current epidemiological situation, computed tomography (CT) of chest organs plays an important role in disease diagnosis. Clinical and CT data allow physicians to quickly establish the probability of the presence and prognosis of patients with coronavirus disease 2019 (COVID-19).

AIMS: This study aimed to predict outcomes in patients with laboratory-confirmed COVID-19 based on chest CT and a semiquantitative visual pulmonary lesion grading system (CT 0-4).

MATERIALS AND METHODS: A retrospective analysis of the Unified Medical Information and Analytical Service and Unified Radiological Information Service records from March 01, 2020 to July 30, 2020 was performed. The inclusion criteria were as follows: patients diagnosed with U07.1 (laboratory-verified coronavirus infection) from March 01, 2020 to July 30, 2020 and referred for a chest CT by a physician with suspected community-acquired pneumonia caused by COVID-19; the maximum period between laboratory verification and CT was not more than five days. The observation period for each patient was at least till 30 days from the date of CT. CT was performed in 48 medical organizations providing primary medical care to adults in Moscow. The exclusion criterion was a negative reverse transcription-polymerase chain reaction results by July 30, 2020. The CT $0-4$ scale is recommended for use in the Russian Federation to estimate the volume of lung parenchyma lesions when COVID-19 is suspected.

RESULTS: The total sample volume was 38,051 patients. In this study, the risk of death was three times higher for CT-4 than for CT- 0 . In the Kaplan-Meier survival curve, the survival rate of patients in the CT-3 category was almost three times lower (hazard ratio $=2.94$ ) than in the CT 0-2 categories; in addition, the higher the initial category of CT, the lower the risk of deterioration. The time for hospitalization decreased with the increase in the CT grade.

CONCLUSION: The visual CT 0-4 scale can be used to predict outcomes, such as hospitalizations and deaths, in patients suspected of COVID-19 who underwent chest CT in primary health care.

Keywords: COVID-19; community-acquired pneumonia; computed tomography.

To cite this article

Morozov SP, Chernina VYu, Blokhin IA, Gombolevskiy VA. Chest computed tomography for outcome prediction in laboratory-confirmed COVID-19: A retrospective analysis of 38,051 cases. Digital Diagnostics. 2020;1(1):27-36. DOI: https://doi.org/10.17816/DD46791 


\section{基于胸部CT的实验室验证COVID-19预后预 测 : 38,051例患者的回顾性分析}

\section{(c) Sergey P. Morozov, Valeria Yu. Chernina, Ivan A. Blokhin, Victor A. Gombolevskiy}

Research and Practical Clinical Center for Diagnostics and Telemedicine Technologies of the Moscow Health Care Department, Moscow, Russian Federation

论证: 在目前的流行病学情况下, 胸部器官CT (胸部器官的计算机断层扫描) 在该病 的诊断中起着重要的作用。临床和CT数据使医生能够快速判断COVID-19患者的存在概 率和预后。

目的：预测实验室证实的COVID-19患者的结果, 基于胸部器官CT, 使用肺实质损伤程 度半定量视觉量表（CT0-CT4量表）。

材料与方法。对2020年3月1日至2020年7月30日期间从统一医疗信息和分析服务处

(UMIAS) 和从统一放射信息服务处（ERIS）卸载的医疗记录和协议进行了回顾性分 析。本研究纳入了根据ICD-10诊断为U07. 1患者的病历（实验室确诊新型冠状病毒感 染病例）。从2020年3月1日至7月30日，这些患者在疑似COVID-19引起的社区获得性 肺炎的内科医生的指导下接受胸部器官CT检查; 实验室检查和胸部器官计算机断层扫 描之间最长允许的时间不超过 5 天。每位病人的随访期由CT日期起计最少为 30 天。这 项研究是在向莫斯科成年人口提供初级医疗保健的 48 个医疗机构中进行的。本研究不 包括截至2020年7月30日COVID-19聚合酶链反应试验结果为阴性的患者。CT0-CT4量表 推荐在俄罗斯联邦用于评估疑似COVID-19病例肺实质损害的程度。

结果。样本量为 38,051 例。根据研究结果, CT-4类患者的死亡风险比CT-0类患者高 3倍。Kaplan-Meyer 生存曲线显示, CT-3类患者的存活比例比CT0-CT2类患者低3倍 $(H R=2.94)$ 。此外, 发现了CT的初始类别越高, 恶化的风险越低。根据胸部器官 CT显示，住院时间随类别的增加而减少。

结果。CT0-CT4的视觉尺度可用于预测疑似COVID-19患者的预后（住院和死亡），如 果患者在初级卫生保健的基础上接受了胸部器官CT检查。

关键词：COVID-19; 社区获得性肺炎; 计算机断层扫描

\section{引用本文:}

Morozov SP, Chernina VYu, Blokhin IA, Gombolevskiy VA. 基于胸部CT的实验室验证COVID-19预后预测: 38,051例患者的回顾性分析. Digital Diagnostics. 2020;1(1):27-36. DOI: https://doi.org/10.17816/DD46791 


\section{ОБОСНОВАНИЕ}

11 марта 2020 г. Всемирная организация здравоохранения объявила пандемию по заболеванию COVID-19, вызываемому вирусом SARS-CoV-2 [1]. По официальным данным, к третьему кварталу 2020 г. в мире зарегистрировано более 29 млн подтверждённых случаев заболевания и более 940 тыс. летальных исходов [2].

В условиях сложившейся эпидемиологической ситуации компьютерная томография органов грудной клетки (КТ ОГК) играет важную роль в диагностике заболевания. Клинические и КТ-данные позволяют врачам в короткие сроки установить вероятность наличия и прогноз у пациентов с COVID-19 [3]. Следует отметить, что при КТ ОГК отсутствуют патогномоничные признаки коронавирусной инфекции, однако наиболее часто визуализируются двусторонние периферические участки «матового стекла», преимущественно в базальных отделах лёгких $[4,5]$. Количественный анализ изменений на КT ОГК облегчает оценку состояния больных COVID-19 [6]. Так, в работе D. Colombi и соавт. [7] представлена количественная оценка поражения лёгочной паренхимы с помощью открытого программного обеспечения, которая установила высокую корреляцию между сохранной, хорошо вентилируемой лёгочной тканью и исходами (перевод в палату интенсивной терапии или смерть). Степень поражения лёгких при COVID-19 можно оценить полностью автоматически, используя алгоритмы машинного обучения [8].

В связи с эпидемиологической обстановкой возникает необходимость создания и критической оценки прогностических моделей на основе клинических данных [9]. На территории Российской Федерации в условиях большого потока пациентов для быстрой и стандартизованной оценки изменений в лёгких, выявленных при КТ ОГК, рекомендована «эмпирическая» визуальная шкала [10].

Цель исследования - прогнозирование исходов у лабораторно верифицированных больных COVID-19 по данным КТ ОГК с помощью полуколичественной визуальной шкалы степени поражения лёгочной паренхимы.

\section{МАТЕРИАЛ И МЕТОДЫ}

\section{Дизайн исследования}

Настоящее ретроспективное исследование согласовано с независимым этическим комитетом Московского регионального отделения Российского общества рентгенологов и радиологов. Информированного согласия пациентов не требовалось ввиду ретроспективного ди- зайна исследования (отсутствие проспективной части исследования с вмешательством в лечебно-диагностический процесс).

\section{Критерии соответствия}

Выполнен анализ выгрузки историй болезни из Единого медицинского информационного-аналитического сервиса (ЕМИАС) и протоколов из Единого радиологического информационного сервиса (ЕРИС) в период с 01.03.2020 по 30.07.2020 включительно.

Kрuтерии включения: пациенты с диагнозом U07.1 по Международной статистической классификации болезней и проблем, связанных со здоровьем, 10-го пересмотра (лабораторно верифицированная коронавирусная инфекция), которым проведена КТ ОГК по направлению врача-терапевта при подозрении на внебольничную пневмонию, вызванную COVID-19; максимально допустимый срок между лабораторной верификацией и КТ ОГК - не более 5 дней. Срок наблюдения за каждым пациентом составлял не менее 30 сут с момента проведения КТ в поликлинике.

Критерии исключения: пациенты, у которых по данным КТ ОГК не выявлено патологических изменений, характерных для COVID-19; пациенты, которым не произведена оценка по системе KTO-KT4; пациенты с отрицательными результатами теста полимеразной цепной реакции на COVID-19 к 30.07.2020.

Всего из ЕМИАС и ЕРИС было отобрано 240985 пациентов. Из исследования исключены 202934 пациента без лабораторной верификации и данных по категориям шкалы КTO-KT4.

\section{Описание медицинского исследования Оборудование и КТ-протокол}

Исследования выполнены на 48 компьютерных томографах, включающих модели Toshiba Aquilion 64 (Canon, Япония), Toshiba Aquilion CXL (Canon, Япония), General Electric HiSpeed (GE, США). Для всех исследований использовался стандартный протокол: напряжение 120 кB; сила тока — автоматически настраиваемая в зависимости от топограммы; направление сканирования - от диафрагмы к верхушкам лёгких; поле обзора (field of view, FOV) 350 мм; толщина срезов $\leq 1$ мм; фильтр реконструкции (kernel): лёгочный для Toshiba (Canon) FC50/FC51/FC52/FC53, для GE - LUNG. Сканирование выполнялось при задержке дыхания на глубине вдоха.

\section{Оценка данных КТ ОГК}

Первичную оценку КТ ОГК выполняли в ЕРИС врачирентгенологи амбулаторных КТ-центров с опытом 
работы от 8 до 22 лет. Дополнительно все исследования были пересмотрены в ЕРИС дежурными врачами-экспертами Московского референс-центра лучевой диагностики, другое программное обеспечение не использовалось. После финализации каждого первичного протокола в пределах 30 мин эксперт референс-центра с опытом работы в торакальной рентгенологии от 10 лет проводил аудит, при необходимости с коррекцией категории по шкале степени поражения лёгочной паренхимы КТ0-КТ4. Таким образом, категория изменялась сразу, без сохранения первичных данных. Согласно отчётам об аудите, процент расхождений при использовании КТО-КТ4 составил до 5\%.

В Российской Федерации, согласно Временным методическим рекомендациям Российского общества рентгенологов и радиологов и Российской ассоциации специалистов по ультразвуковой диагностике в медицине, в условиях большого потока пациентов для оценки изменений в лёгких, выявленных при КТ ОГК, рекомендована так называемая эмпирическая визуальная шкала. Она основана на визуальной оценке примерного объёма поражённой лёгочной ткани [11]. Данная шкала имеет 5 градаций, начинается с «0», далее с интервалами по 25\%. В Департаменте здравоохранения Москвы используются методические рекомендации, согласно которым оценку тяжести поражения лёгочной паренхимы при COVID-19 следует проводить по проценту поражения лёгкого вне зависимости от семиотической фазы процесса («матовое стекло», «булыжная мостовая», консолидация или их комбинация). Этот параметр оценивается отдельно по каждому лёгкому. Категория изменений определяется по лёгкому с наибольшим поражением (вне зависимости от наличия постоперационных изменений) [10].

\section{Гипотезы исследования}

Для проведения исследования были поставлены следующие вопросы:

1. Имеется ли взаимозависимость между значениями КTO-КT4 у лабораторно верифицированных пациентов и риском летального исхода?

2. Имеется ли взаимозависимость между значениями КTO-КТ4 у лабораторно верифицированных пациентов и выживаемостью?

3. Имеется ли взаимозависимость между временем перехода из одной категории КТО-КТ4 в другую и первоначальной категорией КТО-КТ4 у лабораторно верифицированных пациентов?

4. Имеется ли взаимозависимость между значениями КTO-KT4 у лабораторно верифицированных пациентов и количеством дней от первичного КТ до госпитализации?

\section{Статистический анализ}

В анализ данных были включены все пациенты с лабораторно верифицированным диагнозом COVID-19, для которых имелись валидные данные по датам госпитализации и датам проведения по крайней мере одного КТ-обследования. При этом за исходный уровень и дату оценки КТ принимали дату и результат первого КТ-обследования, где была поставлена оценка: для 36958 пациентов это была первая КТ, для 1049 - вторая КТ, для 41 - третья КT, для 3 - четвёртая КТ.

Для анализа данных по летальным исходам была использована модель логистической регрессии. В качестве факторов модели использовали пол и возраст пациентов, а также категорию по шкале КТ0-КТ4. Для каждого фактора была дана оценка отношения шансов (odds ratio, OR) летального исхода, а также 95\% доверительного интервала (ДИ) для OR.

Для анализа данных типа «время до наступления события (общая выживаемость, время до ухудшения КТ, время от первичного КТ до госпитализации)» были использованы метод Каплана-Мейера, а также регрессия Кокса с включением в качестве факторов модели пола, возраста пациентов и категории тяжести по исходному КТ.

Для зависимой переменной «число госпитализаций» было проведено построение регрессии Пуассона с использованием вышеуказанных факторов в качестве ковариатов. Для каждого фактора была дана оценка скорости наступления события (IRR) и соответствующего 95\% ДИ.

Для данных по общей длительности госпитализации (число дней) и числу дней от даты исходного КТ до лабораторной верификации диагноза использовали многофакторную регрессионную модель с факторами «пол», «возраст» и «категория тяжести по КТ». Для каждого фактора приведены значения регрессионных коэффициентов с указанием $95 \%$ ДИ.

Статистическая обработка была выполнена с помощью программы Stata 14.

\section{РЕЗУЛЬТАТЫ}

\section{Объекты (участники) исследования}

Всего из ЕМИАС и ЕРИС было отобрано 240985 пациентов. Из исследования исключены 202934 пациента без лабораторной верификации и данных по катего- 


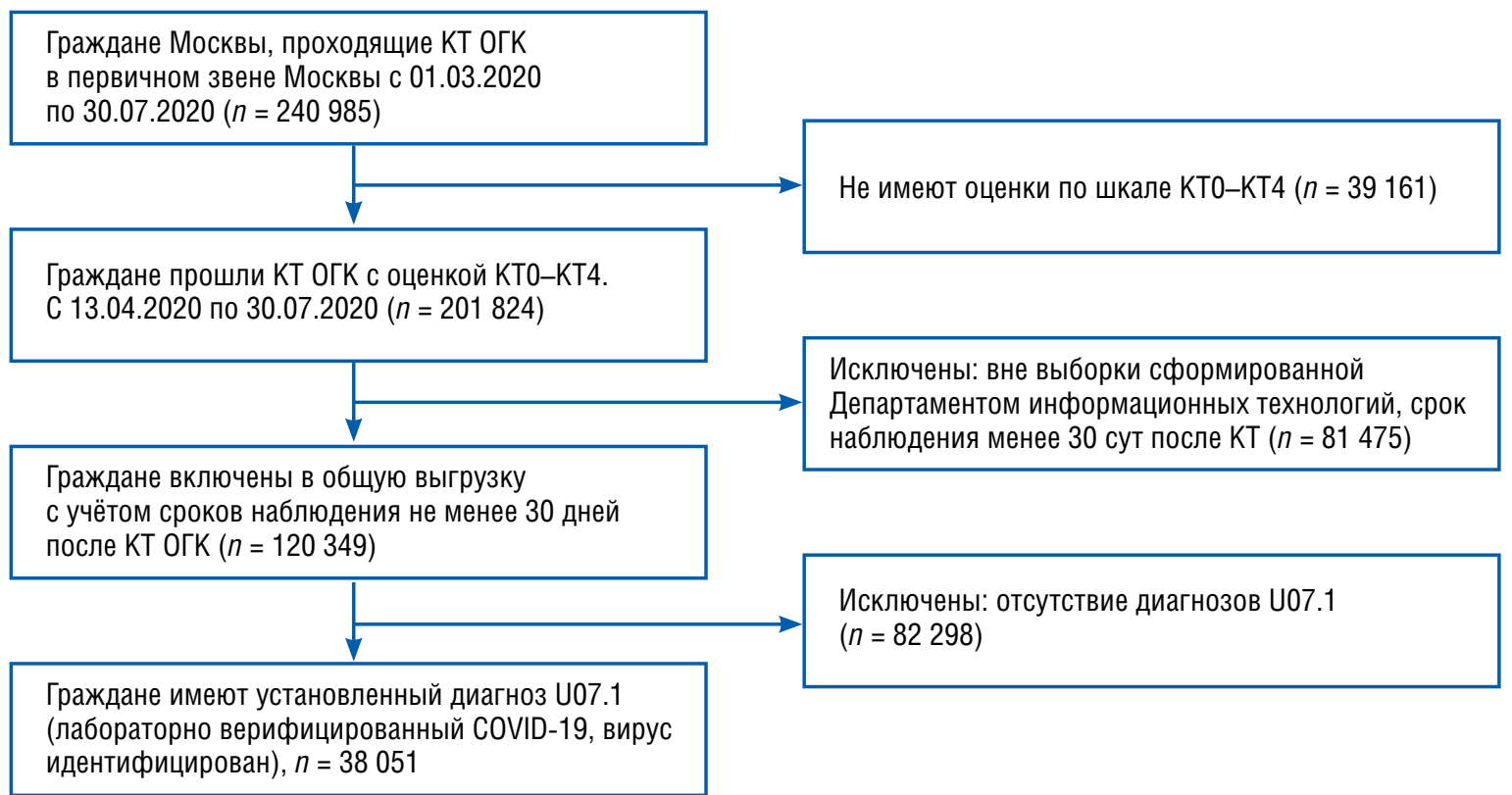

Рис. 1. Блок-схема формирования выборки.

Примечание. КТ ОГК - компьютерная томография органов грудной клетки, КТ0-КТ4 - плуколичественная визуальная шкала степени поражения лёгочной паренхимы.

риям шкалы КТО-КТ4. Итоговый объём выборки 38051 пациент, из них 21888 (57,5\%) мужчин и 16163 $(42,5 \%)$ женщины. Средний возраст - $50 \pm 14,7$ года. Общее количество смертей - 182. Блок-схема формирования выборки представлена на рис. 1. Большинство пациентов при первичном сканировании классифицированы как КТ1 (таблица).

Для пациентов категории KT4 риск смерти был выше в 3 раза $(p=0,010)$. Для категорий КТ2 и КТЗ статистически значимых отличий не выявлено. Аналогичные результаты получены и при анализе общей выживаемости. По кривым Каплана-Мейера для анализа выживаемости доля выживших пациентов в категории КT3 почти в 3 раза ниже (HR $=2,94)$, чем в категориях КTO-КT2 (рис. 2).
Анализ времени до ухудшения состояния по данным КТ ОГК на одну и более категории относительно исходного уровня показал взаимозависимость исходно

Таблица. Распределение пациентов по исходной категории шкалы КТО-КT4

\begin{tabular}{l|c|c}
\hline $\begin{array}{c}\text { Категория } \\
\text { по шкале КТ0-КТ4 }\end{array}$ & $\begin{array}{c}\text { Количество } \\
\text { пациентов, } \boldsymbol{n}\end{array}$ & Доля, \% \\
\hline 0 & 8112 & 21,3 \\
1 & 18704 & 49,2 \\
2 & 8180 & 21,5 \\
3 & 2773 & 7,3 \\
4 & 282 & 0,7 \\
Суммарно & 38051 & 100,0 \\
\hline
\end{tabular}

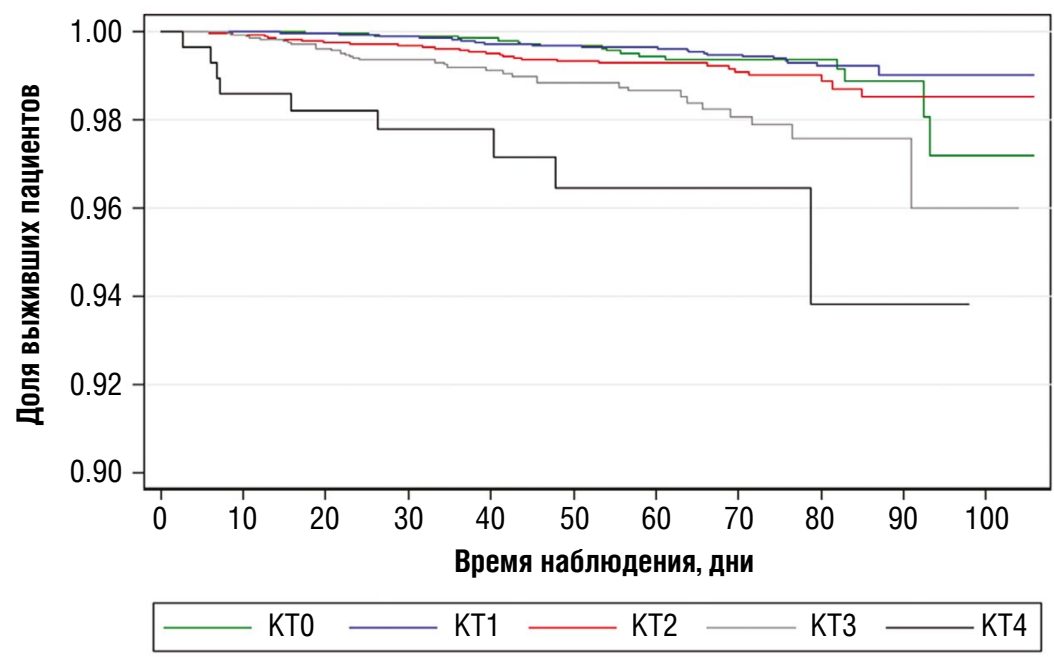

Рис. 2. Кривые общей выживаемости по методу Каплана-Мейера для категорий по шкале КT0-КТ4 ( $p<0,0001)$. 


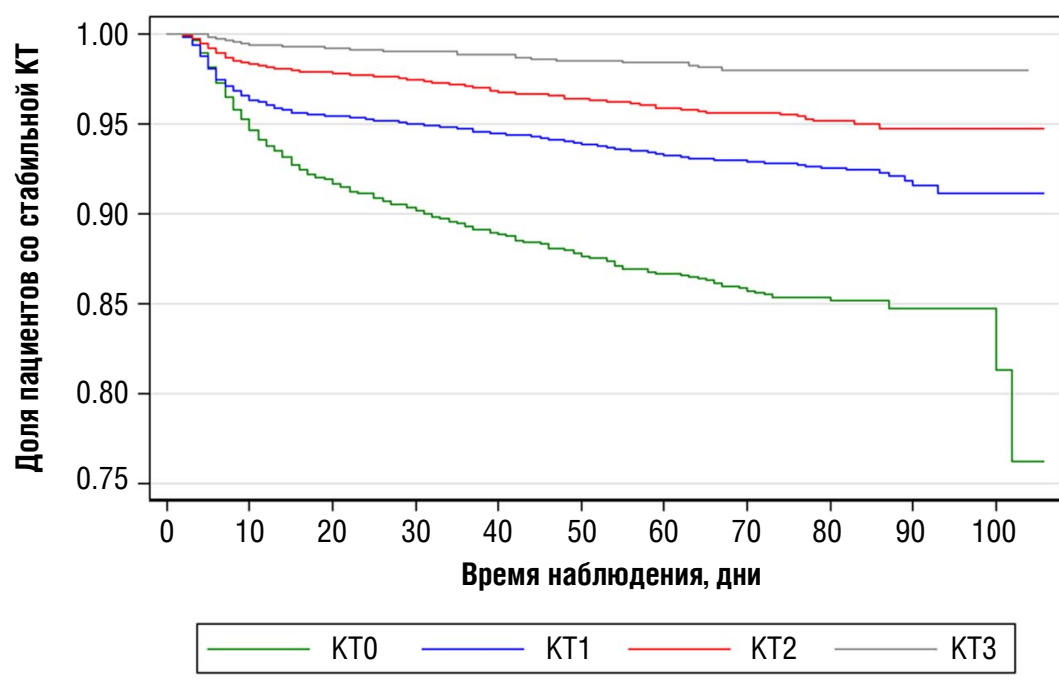

Рис. 3. Кривые Каплана-Мейера для данных по времени до ухудшения состояния по данным компьютерной томографии (КТ) органов грудной клетки на одну и более категории относительно исходного уровня $(p<0,0001)$.

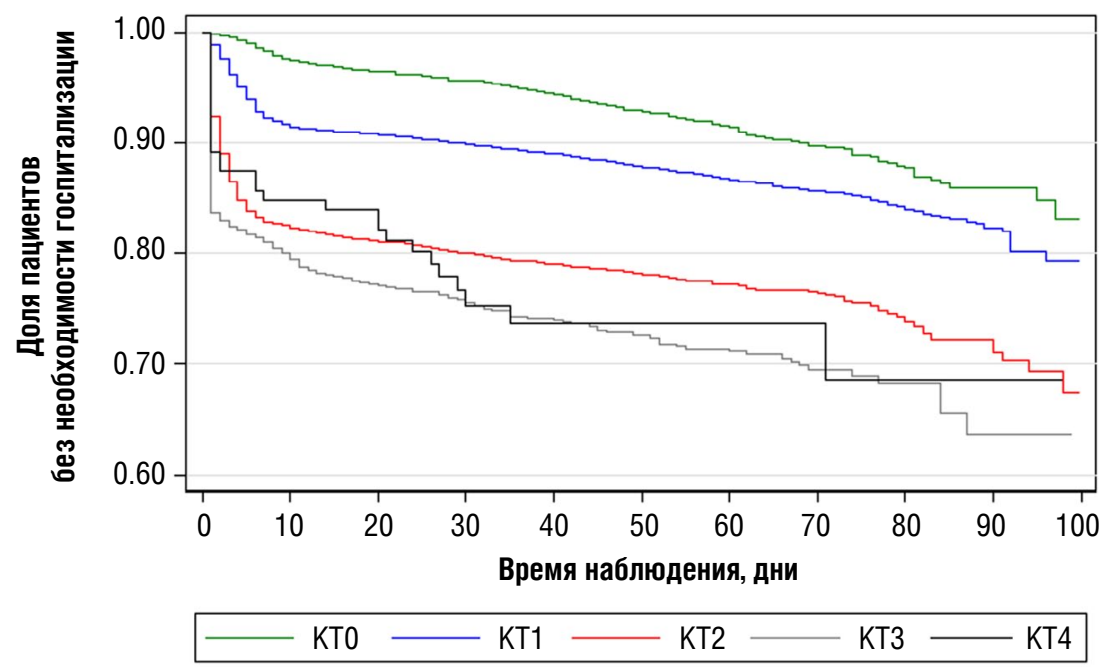

Рис. 4. Кривые Каплана-Мейера для данных по времени от первичной компьютерной томографии (КТ) органов грудной клетки до госпитализации $(p<0,0001)$.

высокого уровня поражения и низкого риска ухудшения $(p<0,001)$ (рис. 3).

Кроме того, было выявлено, что время до госпитализации снижалось при увеличении категории по данным КТ ОГК $(p<0,001) ;$ рис. 4.

При анализе длительности интервала между первой и второй КТ ( $n=12726$ ) среднее время ожидания составило 25,1 $\pm 21,9$ дня (95\% ДИ 24,7-25,5), медианное -20 дней. При анализе длительности интервала между первой и третьей КТ ( $n=2847)$ среднее время ожидания составило $36,6 \pm 28,8$ дня (95\% дИ 35,4$37,5)$, медианное - 30 дней. При анализе длительности интервала между первой и четвёртой КТ ( $n=582)$ среднее время ожидания составило 44,6 $\pm 26,5$ дня (95\% ДИ 42,4-46,7), медианное - 40 дней.

\section{ОБСУЖДЕНИЕ}

По результатам данного исследования выявлено, что для пациентов с категорией КТ4 риск смерти был выше в 3 раза, чем при КТО. По кривым Каплана-Мейера для анализа выживаемости доля выживших пациентов в категории КТ3 была почти в 3 раза ниже $(H R=2,94)$, чем в категориях КTO-KT2. Кроме того, было установлено, что чем выше исходная категория КТ, тем риск ухудшения ниже. На рис. 3 кривая КТЗ является наиболее стабильной во времени, в то время как кривые КТО, КТ1 и КТ2 имеют тенденцию к ухудшению. Именно поэтому лёгкие стадии поражения (КТО, KT1, KT2) требуют такого же внимания, как и тяжёлые, поскольку имеется бо́льший риск прогрессирования заболевания. Время до госпитализации снижалось 
при увеличении категории по данным КТ ОГК. Следует отметить, что решение о госпитализации зависит от клинического статуса пациента, особенностей организации специализированного коечного фонда, нормативно-правовых актов. Пациенты могли быть госпитализированы также вне периода наблюдения или госпитализированы в учреждения, не имеющие подключения к ЕМИАС. Установлен более высокий уровень смертности от всех причин в июне, что может быть связано с загрузкой системы здравоохранения и несовершенством алгоритмов оказания медицинской помощи в условиях сложной эпидемиологической обстановки.

Ранее нами было проведено ретроспективное исследование, по результатам которого выявлено, что вероятность летального исхода направленно увеличивалась от КТО к КТ4. Возраст пациента и категория по шкале КTO-КТ4 были статистически значимо ассоциированы со временем до наступления смерти от COVID-19. При переходе из одной категории КТ в следующую риск увеличивался в среднем на $38 \%$ [12]. Однако в данное исследование были включены пациенты без лабораторной верификации коронавирусной инфекции, период наблюдения за пациентами был значительно меньше, а оценивались только корреляция степени тяжести поражения по КТ ОГК и летальные исходы.

Настоящее исследование дополняет ранее полученные результаты, основано на большей выборке с лабораторно верифицированными диагнозами и более подробной информацией об исходах.

Установлено, что КТ ОГК позволяет выявить изменения в лёгких, характерные для COVID-19, и оценить их степень, что совпадает с результатами исследований других авторов [13, 14]. В работе M. Yuan и соавт. [15] разработана прогностическая модель летальных исходов при COVID-19, учитывающая данные КT, но использующая комплексную посегментную оценку КТ-данных. Многокомпонентная полуколичественная оценка изменений представляется ограничением модели, способным привести к накоплению ошибок. К другим ограничениям модели можно отнести длительное время для анализа данных и сложность использования в рутинной практике. Предлагаемый в нашем исследовании вариант оценки данных КТ ОГК легко применим на практике, коррелирует с риском смерти от всех причин, общей выживаемостью, риском ухудшения клинического статуса.

В публикации С. Петрикова и соавт. [16] выявлена достоверно значимая корреляционная связь между увеличением степени поражения лёгких, определённой с помощью КТ, с ухудшением клинического состояния пациентов. В публикации Р. Хu и соавт. [17] в ходе ретроспективного мультицентрового наблюдательного исследования проведён мультивариантный анализ 703 лабораторно верифицированных случаев COVID-19, показавший корреляцию между летальным исходом и наличием сопутствующих заболеваний, лейкоцитозом, лимфопенией, обширным поражением лёгочной паренхимы по данным КТ. Авторами предложена визуальная посегментная полуколичественная шкала оценки поражения лёгких, где поражённый сегмент оценивается в 1 балл вне зависимости от морфологии инфильтративных изменений, при вовлечении 14 (70\%) сегментов и более риск летального исхода увеличивается в 3 раза. B работе D. Colombi и соавт. [7] представлена количественная оценка поражения лёгочной паренхимы с помощью открытого программного обеспечения, которая установила высокую корреляцию между сохранной, хорошо вентилируемой лёгочной тканью и исходами (перевод в палату интенсивной терапии или смерть). В работе Y. Xiong и соавт. [18] на маленькой выборке из 42 пациентов отмечена положительная корреляция между количеством поражённых долей лёгких при первичном исследовании и риском увеличения площади инфильтративных изменений. Результаты этих исследований сопоставимы с нашими. Основные отличия заключаются в использовании оригинальной шкалы KTO-KT4, а также в обследованных контингентах: в нашем случае это только пациенты, самостоятельно обратившиеся за медицинской помощью в первичное звено здравоохранения.

\section{Ограничения исследования}

Наше исследование имеет ряд ограничений. Вопервых, анализ данных проведён ретроспективно. Однако такой дизайн позволил включить в исследование большое количество пациентов с большим периодом наблюдения. Во-вторых, авторами статьи не производился пересмотр исследований КТ ОГК, что могло повлиять на распределение пациентов по категориям шкалы КTO-КT4. Учитывая большой размер выборки, влияние пограничных случаев оценки с занижением или завышением степени поражения лёгочной паренхимы было минимизировано. Кроме того, все исследования были пересмотрены дежурными врачами-экспертами Московского референс-центра лучевой диагностики. В-третьих, использование большого массива данных, сформи- 
рованных полуавтоматическим методом, ограничивает возможности по валидации. Тем не менее высокие уровни статистической значимости и результаты литературного анализа подкрепляют валидность полученных результатов настоящего исследования.

\section{ЗАКЛЮЧЕНИЕ}

У пациентов, которым при подозрении на COVID-19 выполнена КТ ОГК на базе первичного звена здравоохранения, визуальная шкала КTO-КT4 может быть использована в качестве предиктора исходов (госпитализаций и летальных исходов).

\section{СПИСОК ЛИТЕРАТУРЫ}

1. World Health Organization. Timeline of WHO's response to COVID-19 [Internet]. WHO, 2020 [дата обращения: 09.09.2020]. Доступ по ссылке: https://www.who.int/news-room/detail/2906-2020-covidtimeline

2. Dong E., Du H., Gardner L. An interactive web-based dashboard to track COVID-19 in real time // Lancet Infect Dis. 2020. Vol. 20, N 5. P. 533-534. doi: 10.1016/S1473-3099(20)30120-1

3. Zhang R., Ouyang H., Fu L., et al. CT features of SARS-CoV-2 pneumonia according to clinical presentation: a retrospective analysis of 120 consecutive patients from Wuhan city // Eur Radiol. 2020. Vol. 30, N 8. P. 4417-4426. doi: 10.1007/s00330-020-06854-1

4. Silverstein W.K., Stroud L., Cleghorn G.E., Leis J.A. First imported case of 2019 novel coronavirus in Canada, presenting as mild pneumonia // The Lancet. 2020. Vol. 395, N 10225. P. 734. doi: 10.1016/S0140-6736(20)30370-6

5. Yoon S.H., Lee K.H., Kim J.Y., et al. Chest radiographic and CT findings of the 2019 Novel Coronavirus Disease (COVID-19): analysis of nine patients treated in Korea // Korean J Radiol. 2020. Vol. 21, N 4. P. 494-500. doi: 10.3348/kjr.2020.0132

6. Sverzellati N., Milanese G., Milone F., et al. Integrated radiologic algorithm for COVID-19 pandemic // J Thorac Imaging. 2020. Vol. 35, N 4. P. 228-233. doi: 10.1097/RTI.0000000000000516

7. Colombi D., Bodini F.C., Petrini M., et al. Well-aerated lung on admitting chest $\mathrm{CT}$ to predict adverse outcome in $\mathrm{CO}$ VID-19 pneumonia // Radiology. 2020. Vol. 296, N 2. E86-E96. doi: 10.1148/radiol.2020201433

8. Li K., Fang Y., Li W., et al. CT image visual quantitative evaluation and clinical classification of coronavirus disease (COVID-19) // Eur Radiol. 2020. Vol. 30, N 8. P. 4407-4416. doi: 10.1007/s00330-020-06817-6

9. Wynants L., van Calster B., Collins G.S., et al. Prediction models for diagnosis and prognosis of covid-19: systematic review and critical appraisal // BMJ. 2020. Vol. 369. M1328. doi: 10.1136/bmj.m1328 10. Морозов С.П., Проценко Д.Н., Сметанина С.В. и др. Лучевая диагностика коронавирусной болезни (COVID-19): органи-

\section{ДОПОЛНИТЕЛЬНО}

Источник финансирования. Исследование и публикации статьи осуществлены на личные средства авторского коллектива.

Конфликт интересов. Авторы данной статьи подтвердили отсутствие конфликта интересов, о котором необходимо сообщить.

Участие авторов. С.П. Морозов - концепция исследования, утверждение финальной версии рукописи; В.Ю. Чернина поиск публикаций по теме статьи, редактирование текста рукописи; И.А. Блохин - формирование набора данных, написание текста рукописи; В.А. Гомболевский - экспертная оценка информации, утверждение финальной версии рукописи. Все авторы внесли существенный вклад в проведение исследования и подготовку статьи, прочли и одобрили финальную версию до публикации.

зация, методология, интерпретация результатов : препринт № ЦДТ - 2020 - II. Версия 2 от 17.04.2020. Серия «Лучшие практики лучевой и инструментальной диагностики». Вып. 65. Москва : ГБУЗ «НПКЦ ДиТ ДЗМ», 2020. 78 с.

11. Синицын В.Е., Тюрин И.Е., Митьков В.В. Временные методические рекомендации Российского общества рентгенологов и радиологов (РОРР) и Российской ассоциации специалистов ультразвуковой диагностики в медицине (РАСУДМ) «Методы лучевой диагностики пневмонии при новой коронавирусной инфекции при COVID-19» (версия 2) // Вестник рентгенологии и радиологии. 2020. Т. 101, № 2. С. 72-89. doi: 10.20862/0042-4676-2020-101-2-72-89

12. Морозов С.П., Гомболевский В.А., Чернина В.Ю. и др. Прогнозирование летальных исходов при COVID-19 по данным компьютерной томографии органов грудной клетки // Туберкулез и болезни легких. 2020. Т. 98, № 6. С. 7-14. doi: 10.21292/2075-1230-2020-98-6-7-14

13. Христенко Е.А., фон Стакельберг О., Кауцор Х.У. и др. КТпаттерны при COVID-19 ассоциированных пневмониях стандартизация описаний исследований на основе глоссария общества Флейшнера // REJR. 2020. T. 10, № 1. С. 16-26. doi: 10.21569/2222-7415-2020-10-1-16-26

14. Raptis C.A., Hammer M.M., Short R.G., et al. Chest CT and coronavirus disease (COVID-19): a critical review of the literature to date // AJR Am J Roentgenol. 2020. Vol. 215, N 4. P. 839-842. doi: 10.2214/AJR.20.23202

15. Yuan M., Yin W., Tao Z., et al. Association of radiologic findings with mortality of patients infected with 2019 novel coronavirus in Wuhan, China // PLoS One. 2020. Vol. 15, N 3. E0230548. doi: 10.1371/journal.pone.0230548

16. Петриков С.С., Попугаев К.А., Бармина Т.Г. и др. Сопоставление клинических данных и компьютерно-томографической семиотики легких при COVID-19 // Tyберкулез и болезни легких. 2020. Т. 98, № 7. С. 14-25. doi: 10.21292/2075-1230-2020-98-7-14-25 
17. Xu P.P., Tian R.H., Luo S., et al. Risk factors for adverse clinical outcomes with COVID-19 in China: a multicenter, retrospective, observational study // Theranostics. 2020. Vol. 10, N 14. P. 6372 6383. doi: 10.7150/thno.46833

\section{REFERENCES}

1. World Health Organization. Timeline of WHO's response to COVID-19 [Internet]. WHO; 2020 [cited 2020 Sept 9]. Available from: https://www.who.int/news-room/detail/29-06-2020-covidtimeline

2. Dong E, Du H, Gardner L. An interactive web-based dashboard to track COVID-19 in real time. Lancet Infect Dis. 2020;20(5):533534. doi: 10.1016/S1473-3099(20)30120-1

3. Zhang R, Ouyang $\mathrm{H}$, Fu L, et al. CT features of SARS-CoV-2 pneumonia according to clinical presentation: a retrospective analysis of 120 consecutive patients from Wuhan city. Eur Radiol. 2020;30(8):4417-4426. doi: 10.1007/s00330-020-06854-1

4. Silverstein WK, Stroud L, Cleghorn GE, Leis JA. First imported case of 2019 novel coronavirus in Canada, presenting as mild pneumonia. Lancet. 2020;395(10225):734. doi: 10.1016/S0140-6736(20)30370-6

5. Yoon SH, Lee KH, Kim JY, et al. Chest radiographic and CT findings of the 2019 Novel Coronavirus Disease (COVID-19): analysis of nine patients treated in Korea. Korean J Radiol. 2020;21(4):494500. doi: 10.3348/kjr.2020.0132

6. Sverzellati N, Milanese G, Milone F, et al. Integrated radiologic algorithm for COVID-19 pandemic. J Thorac Imaging. 2020;35(4):228-233. doi: 10.1097/RTI.0000000000000516

7. Colombi D, Bodini FC, Petrini $M$, et al. Well-aerated lung on admitting chest CT to predict adverse outcome in COVID-19 pneumonia. Radiology. 2020;296(2):E86-E96. doi: 10.1148/radiol.2020201433

8. LiK, Fang Y, Li W, et al. CT image visual quantitative evaluation and clinical classification of coronavirus disease (COVID-19). Eur Radiol. 2020;30(8):4407-4416. doi: 10.1007/s00330-020-06817-6

9. Wynants $L$, van Calster B, Collins GS, et al. Prediction models for diagnosis and prognosis of covid-19: systematic review and critical appraisal. BMJ. 2020;369:M1328. doi: 10.1136/bmj.m1328

10. Morozov SP, Protsenko DN, Smetanina SV, et al. Radiation diagnostics of coronavirus disease (COVID-19): organization, methodology, interpretation of results: Preprint No. CDT - 2020 - II. Version 2 from 17.04.2020. Series «Best practices of radiation and
18. Xiong Y., Sun D., Liu Y., et al. Clinical and High-Resolution CT Features of the COVID-19 Infection: Comparison of the Initial and Follow-up Changes // Investigative Radiology. 2020. Vol. 55, N 6. P. 332-339. doi: 10.1097/RLI.0000000000000674 instrumental diagnostics». Issue 65. Moscow: GBUZ «NPKTS DIT DZM»; 2020. 78 p. (In Russ).

11. Sinitsyn VE, Tyurin IE, MitkovVV.Consensus Guidelines of Russian Society of Radiology (RSR) and Russian Association of Specialists in Ultrasound Diagnostics in Medicine (RASUDM) «Role of Imaging ( $X$ ray, CT and US) in Diagnosis of COVID-19 Pneumonia» (version 2). Journal of radiology and nuclear medicine. 2020;101(2):72-89. (In Russ). doi: 10.20862/0042-4676-2020-101-2-72-89

12. Morozov SP, Gombolevskiy VA, Chernina VY, et al. Prediction of lethal outcomes in COVID-19 cases based on the results chest computed tomography. Tuberculosis and Lung Diseases. 2020;98(6):7-14. (In Russ). doi: 10.21292/2075-1230-2020-98-6-7-14

13. Khristenko E, von Stackelberg 0 , Kauczor HU, et al. Ctpatterns in COVID-19 associated pneumonia - unification of radiological reports based on glossary of Fleischner society. REJR. 2020;10(1):1626. (In Russ). doi: 10.21569/2222-7415-2020-10-1-16-26

14. Raptis CA, Hammer MM, Short RG, et al. Chest CT and coronavirus disease (COVID-19): a critical review of the literature to date. AJR Am J Roentgenol. 2020;215(4):839-842. doi: 10.2214/AJR.20.23202

15. Yuan M, Yin W, Tao Z, et al. Association of radiologic findings with mortality of patients infected with 2019 novel coronavirus in Wuhan, China. PLoS One. 2020;15(3):E0230548. doi: 10.1371/journal.pone.0230548

16. Petrikov SS, Popugaev KA, Barmina TG, et al. Comparison of clinical data and computed tomography semiotics of the lungs in COVID-19. Tuberculosis and Lung Diseases. 2020;98(7):14-25. (In Russ). doi: 10.21292/2075-1230-2020-98-7-14-25

17. Xu PP, Tian RH, Luo S, et al. Risk factors for adverse clinical outcomes with COVID-19 in China: a multicenter, retrospective, observational study. Theranostics. 2020;10(14):6372-6383. doi: 10.7150/thno.46833

18. Xiong $Y$, Sun D, Liu Y, et al. Clinical and High-Resolution CT Features of the COVID-19 Infection: Comparison of the Initial and Follow-up Changes. Invest Radiol. 2020;55(6):332-339. doi: 10.1097/RLI.0000000000000674

\section{ОБ АВTOPAX}

*Гомболевский Виктор Александрович, К.М.Н.; адрес: Россия, 127051, Москва, улица Петровка, д.24/1; ORCID: https://orcid.org/0000-0003-1816-1315; eLibrary SPIN: 6810-3279; e-mail: g_victor@mail.ru

Морозов Сергей Павлович, д-р мед. наук, профессор; ORCID: http://orcid.org/0000-0001-6545-6170; eLibrary SPIN: 8542-1720; e-mail: morozov@npcmr.ru

\section{Чернина Валерия Юрьевна;}

ORCID: http://orcid.org/0000-0002-0302-293X; eLibrary SPIN: 8896-8051; e-mail: v.chernina@npcmr.ru

\section{Блохин Иван Андреевич;}

ORCID: http://orcid.org/0000-0002-2681-9378; eLibrary SPIN: 3306-1387; e-mail: i.blokhin@npcmr.ru

\section{AUTHORS INFO}

*Victor A. Gombolevskiy, MD, PhD, MPH; address: Petrovka 24/1, Moscow, Russia, 127051; ORCID: https://orcid.org/0000-0003-1816-1315; eLibrary SPIN: 6810-3279; e-mail: g_victor@mail.ru

Sergey P. Morozov, MD, PhD, Professor; ORCID: http://orcid.org/0000-0001-6545-6170; eLibrary SPIN: 8542-1720; e-mail: morozov@npcmr.ru

Valeria Yu. Chernina, MD;

ORCID: http://orcid.org/0000-0002-0302-293X; eLibrary SPIN: 8896-8051; e-mail: v.chernina@npcmr.ru

Ivan A. Blokhin, MD;

ORCID: http://orcid.org/0000-0002-2681-9378; eLibrary SPIN: 3306-1387; e-mail: i.blokhin@npcmr.ru 